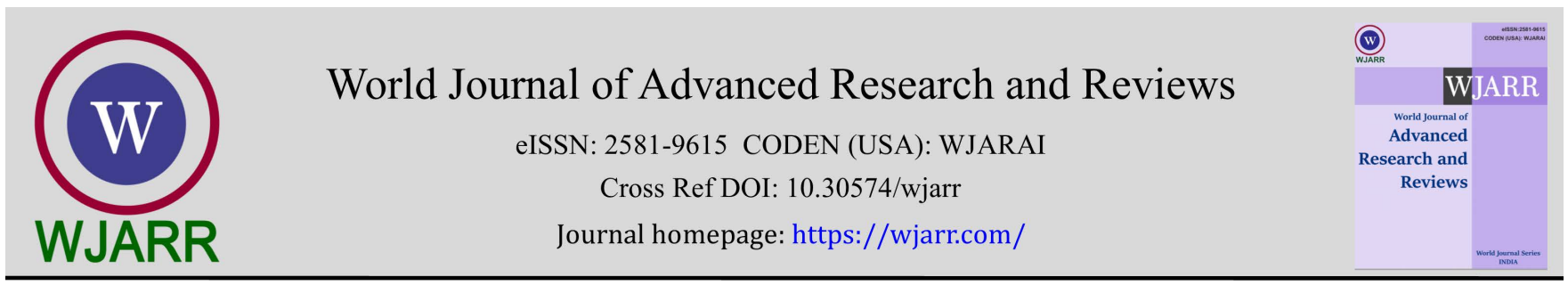

(RESEARCH ARTiClE)

Check for updates

\title{
Significant changes in Complete Blood Count (CBC) and blood coagulation in SARS- CoV-2 infection: A retrospective study
}

Salma Rouhi 1, 2, *, Hisham Yahayaoui 1, 2, Zineddine Tahouri 1,2, Saloua Abbassi 1,2, Mustapha AitAmeur 1,2 and Mohammed Chakour ${ }^{1,2}$

${ }^{1}$ Laboratory of Hematology, Avicenna Military Hospital of Marrakech. Morocco.

2 Faculty of medicine and pharmacy, Cadi Ayyad University. Marrakech. Morocco.

World Journal of Advanced Research and Reviews, 2022, 13(02), 476-482

Publication history: Received on 15 January 2022; revised on 16 February 2022; accepted on 18 February 2022

Article DOI: https://doi.org/10.30574/wjarr.2022.13.2.0168

\begin{abstract}
Introduction: The aim of this work is to explore all the abnormalities of the hemogram and hemostasis tests in patients with severe COVID disease.

Methods: A cohort of patients with COVID-19 admitted to Avicenna military hospital in Marrakech during the 6 months from April to October 2020 was retrospectively analyzed. The baseline data of laboratory tests, including complete blood count (CBC) and coagulation tests.
\end{abstract}

Results: A total of 218 individuals were enrolled, with 82 patients meeting the criteria for severe covid and admitted to the intensive care unit, and 136 considered stable and admitted to the medicine departments.

The average age of patients with severe covid is significantly higher than non-severe patients (65.21 \pm 5.71 versus 49.13 $\pm 7.81, \mathrm{p}=0.038$ ).

Between the groups with severe and non-severe COVID-19: only platelets, lymphocytes and D-dimer showed statically significant difference ( $\mathrm{P}=0.0001,0.003$ and 0.0001 respectively). But the differences were relatively small for the other parameters.

The calculated parameters NLR, d-NLR, and LMR did not differ statistically between the two study groups with the exception of PLR, which was positively correlated with the severity of COVID $(P=0,007)$.

The ROC analysis showed that the best area under the curve (AUC) was obtained with platelet rate, D-dimer and PLR (0.86, 0.73 and 0.69 respectively) with the best cut-offs $(250 \mathrm{~g} / \mathrm{L}, 0.5 \mu \mathrm{g} / \mathrm{l}$ and 300 correspondingly).

Conclusion: Thrombocytopenia, lymphopenia, increased PLR and D-dimers are often encountered during severe Covid and can be used as a predictive marker for the severity of the disease.

Keywords: Sars; Cov2; Neutrophils; Lymphocytes; Coagulation

\footnotetext{
${ }^{*}$ Corresponding author: Salma Rouhi

Laboratory of Hematology, Avicenna Military Hospital of Marrakech;Morocco.

Copyright $(2022$ Author(s) retain the copyright of this article. This article is published under the terms of the Creative Commons Attribution Liscense 4.0.
} 


\section{Introduction}

The identification of clinical and laboratory markers of development to severe and lethal forms is critical in the fight against corona virus 2019 (COVID-19) illness. These indicators will aid in identifying patients who are at a high risk of acquiring severe covid and predicting their prognosis.

These predictions will help with risk stratification, guiding interventional research to patients at higher risk of acquiring severe disease, and maximizing the use of limited human and technical resources in the current epidemic. Furthermore, identifying laboratory measures capable of distinguishing between severe and non-severe cases, as well as those with a high or low risk of death, will allow for improved clinical situational awareness [1].

Early reports from China identified an exaggerated inflammatory response (cytokine storm) as one of the distinct features of SARS-CoV-2 infection, at least in a subset of patients [2]. Since then, significant elevations in serum C-reactive protein (CRP), interleukin 6 (IL-6), ferritin, procalcitonin, fibrinogen, and other acute phase reactants have been frequently observed in patients with severe COVID-19 [3].

Lymphopenia was singled out as a hallmark of COVID-19 when the first descriptive studies from Wuhan showed that over $80 \%$ of patients with COVID-19 had low lymphocyte counts [4]. Other changes have been found on CBC: such as leukopenia, eosinopenia, monocytosis, neutrophilia, and thrombocytopenia. The severity of lymphopenia and thrombocytopenia in COVID-19 patients has been linked to more serious outcomes, such as pneumonia, ARDS, organ failure and mortality [5].

The authors suggested using other indicators of inflammation: peripheral white blood cell (WBC) count, neutrophil (NEU)-to-lymphocyte (LYM) ratio (NLR), derived NLR ratio (d-NLR, neutrophil count divided by the result of WBC count minus neutrophil count), platelet-to-lymphocyte ratio (PLR), and lymphocyte-to-monocyte ratio (LMR), as surrogate markers of disease severity in COVID-19 [5-7].

additionally, a subset of patients with COVID-19 have alterations in coagulation factors manifesting as slightly prolonged prothrombin times (PT) and increased D-dimer levels, with some COVID-19 patients presenting with coagulopathy and disseminated intravascular coagulation [8].

The aim of this work is to compare detailed CBC differences and coagulation parameters between severe and nonsevere COVID-19 patients to enable risk stratification and improve clinical situational awareness.

\section{Material and methods}

\subsection{Patients}

For this retrospective cohort study, COVID-19 patients who were admitted to Avicenna military hospital in Marrakech during the 6 months from April to October 2020, and confirmed by a positive real-time reverse transcriptasepolymerase chain reaction assay for nasal and pharyngeal swab specimens, were consecutively included.

Patients were divided into 2 groups: non severe patients and severe patients who have at least one of the following conditions:

- Shortness of breath, $\mathrm{RR} \geq 30$ times/min

- Oxygen saturation (Resting state) $\leq 93 \%$

- $\mathrm{PaO}_{2} / \mathrm{FiO}_{2} \leq 300 \mathrm{mmHg}$.

The imperative of informed consent was waived in light of the anonymous, retrospective, and observational character of this study.

\subsection{Laboratory data}

The complete blood count analysis was carried out on Sysmex XT4000 system, while Blood coagulation tests including plasma D-dimer, prothrombin time (PT), activated partial thromboplastin time (APTT) were measured STA compact from Stago. 
Neutrophil/lymphocyte, lymphocytes/monocytes as well as platelet/lymphocyte ratios were calculated in accordance with the CBC results.

\subsection{Statistics}

Summary statistics of the demographic and clinical characteristics of all patients were expressed as frequencies and proportions for categorical variables, mean \pm SD or median and interquartile for continuous variables. The optimal cutoff values of the continuous values were calculated by applying the receiver operating curve (ROC) analysis.

$\mathrm{P}<0.05$ was recognized as statistically significant. All these statistical calculations were performed using the SPSS 25.0 software (SPSS Inc, Chicago, USA).

\section{Results}

A total of 218 patients were recruited, 82 of whom met the criteria for severe covid and were hospitalized in the intensive care unit, and 136 patients were considered stable and hospitalized in the internal medicine department.

The clinical and biological characteristics of the 2 severe and non-severe covid groups have been described in Table 1 .

Table 1 Baseline characteristics and results of $\mathrm{CBC}$ and coagulation test

\begin{tabular}{|l|c|c|c|}
\hline & severe COVID & non-severe COVID & $\boldsymbol{P}$ \\
\hline Age & $65.21 \pm 5.71$ & $49.13 \pm 7.81$ & 0.038 \\
\hline WBC & $12.12 \pm 6.37$ & $11.19 \pm 6.12$ & 0.28 \\
\hline Neutrophils & $9.59 \pm 6.33$ & $9.25 \pm 5.72$ & 0.69 \\
\hline Eosinophils & $0.9 \pm 0.17$ & $0.06 \pm 0.09$ & 0.176 \\
\hline Basophils & $0.02 \pm 0.01$ & $0.02 \pm 0.02$ & 0.128 \\
\hline Lymphocytes & $1.40 \pm 0.97$ & $1.03 \pm 0.64$ & 0.003 \\
\hline Platelets & $158.13 \pm 77.59$ & $296.87 \pm 102.77$ & 0.0001 \\
\hline Hemoglobin & $12.69 \pm 2.55$ & $11.84 \pm 3.74$ & 0.042 \\
\hline Hematocrit & $37.08 \pm 6.23$ & $35.19 \pm 9.21$ & 0.076 \\
\hline RBC & $4.52 \pm 3.40$ & $4,19 \pm 1.12$ & 0.442 \\
\hline NLR & $21.91 \pm 71.52$ & $12.57 \pm 14.14$ & 0.146 \\
\hline dNLR & $6.27 \pm 4.82$ & $5.61 \pm 4.72$ & 0.335 \\
\hline PLR & $334.03 \pm 279.90$ & $231.46 \pm 235.07$ & 0.007 \\
\hline LMR & $2.11 \pm 1.53$ & $1.95 \pm 1.74$ & 0.449 \\
\hline PT & $68.90 \pm 26.85$ & $80.59 \pm 19.23$ & 0.009 \\
\hline APTT & $43.03 \pm 19.78$ & $48.43 \pm 13.44$ & 0.13 \\
\hline D-Dimers & $5.49 \pm 11.77$ & $17.13 \pm 19.41$ & 0.0001 \\
\hline fibrinogen & $4.87 \pm 1.90$ & $4.09 \pm 1.70$ & 0.22 \\
\hline
\end{tabular}

The average age of patients with severe covid is significantly greater than non-severe patients $(65.21 \pm 5.71$ versus $49.13 \pm 7.81, \mathrm{p}=0.038)$

Between the groups with severe and non-severe COVID-19, there were some significant variations in basic CBC values and blood coagulation tests. A number of parameters showed differences between groups that reached statistical significance, such as platelets, lymphocytes, and d-dimer $(P=0.0001,0.003$ and 0.0001 respectively). But for many of these (including hemoglobin, hematocrit, WBC, Neutrophils), the differences were relatively small.

Table 2 . 
Table 2 Areas under the curves of $\mathrm{CBC}$ and coagulation parameters

\begin{tabular}{|l|c|c|c|c|c|c|}
\hline & AUC & cut-off & sensibility & Specificity & $\begin{array}{c}\text { asymptomatic 95\% } \\
\text { confidence interval }\end{array}$ & $\begin{array}{c}\text { asymptomatic } \\
\text { sigmoid }\end{array}$ \\
\hline platelets & 0.86 & 250000 & 0.84 & 0.4 & $0.78-0.95$ & $<0.0001$ \\
\hline lymphocytes & 0.61 & 1000 & 0.76 & 0.66 & $0.25-0.68$ & 0.008 \\
\hline Neutrophils & 0.62 & 7250 & 0.68 & 0.48 & $0.45-0.80$ & 0.15 \\
\hline NLR & 0.57 & 10.33 & 0.68 & 0.52 & $0.40-0.73$ & 0.46 \\
\hline dNLR & 0.5 & 6.45 & 0.5 & 0.48 & $0.32-0.68$ & 0.96 \\
\hline PLR & 0.69 & 300 & 0.74 & 0.41 & $0.58-0.80$ & $<0.0001$ \\
\hline LMR & 0.51 & 1.97 & 0.76 & 0.73 & $0.39-0.63$ & 0.79 \\
\hline D-dimer & 0.73 & 0.5 & 0.85 & 0.72 & $0.57-0.89$ & 0.004 \\
\hline PT & 0.62 & 0.8 & 0.61 & 0.43 & $0.50-0.74$ & 0.038 \\
\hline
\end{tabular}

The calculated parameters NLR, d-NLR, and LMR did not differ statistically between the two study groups with the exception of PLR, which was positively correlated with the severity of COVID $(p=0,007)$

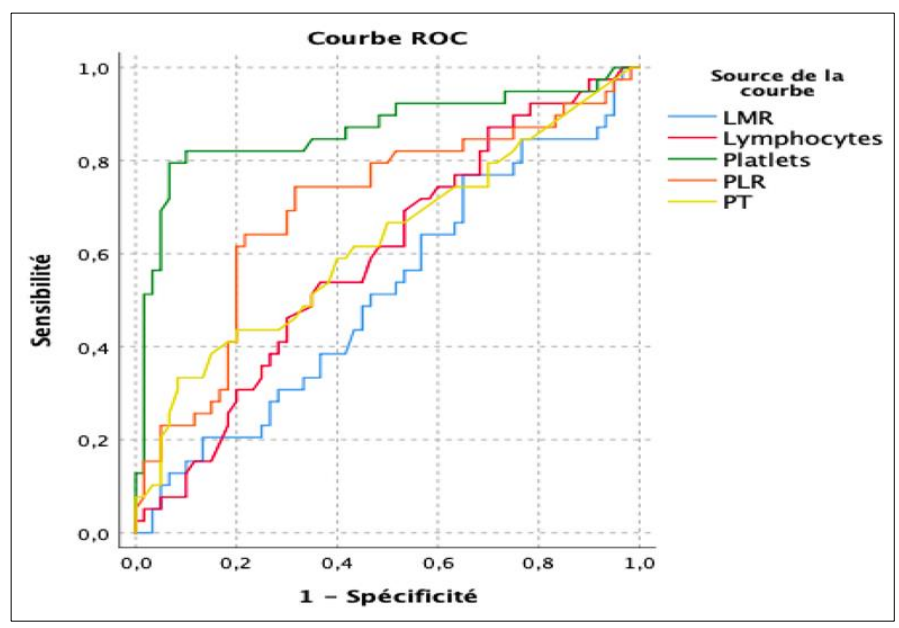

Figure 1 ROC curve used to distinguish patients with severe and non-severe COVID

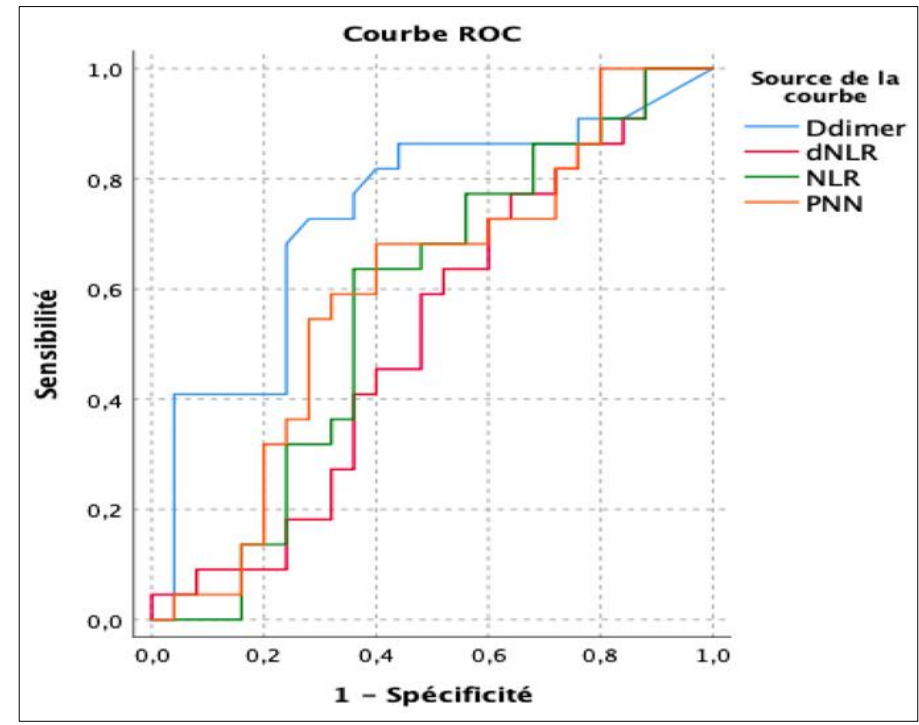


Figure 2 ROC curve used to distinguish patients with severe and non-severe COVID

Given that neutrophils, lymphocytes, platelets, NLR, LMR, PLR, d-NLR, and D-dimer were used to identify patients with severe or non-severe cases of COVID-19, we analyzed the optimal cut-off values calculated by the ROC analysis: The best area under the curve (AUC) was obtained with platelet rate, D-dimer and PLR $(0.86,0.73$ and 0.69 respectively) with the best cut-offs ( $250 \mathrm{~g} / \mathrm{L}, 0.5 \mu \mathrm{g} / \mathrm{l}$ and 300 correspondingly). Figure 1 and

Figure 2.

\section{Discussion}

In this study, a distinct pattern of inflammatory, hematologic, and hemostatic biomarker abnormalities was evaluated in patients with and without severe disease, suggesting that these biomarkers could be used to make significant distinctions in risk stratification models.

Our findings suggest that biological test changes seen in COVID-19 are related to disease severity [5] but also suggest that these alterations may not be restricted to COVID-19-specific pathogenic pathways [9].

Regarding CB counts, severe COVID patients were overall more likely to be thrombopenic (the average of platelets was $158.13 \pm 77.59$ vs $296.87 \pm 102.77 \mathrm{P}=0.001$ ) and lymphopenic (the average of lymphocytes was $1.03 \pm 0.64$ vs $1.40 \pm 0.97 \mathrm{p}=0.003$ ) compared to those with non-severe covid.

The magnitude of lymphopenia and thrombocytopenia in patients with COVID-19 has been associated with more severe outcomes including admission to the intensive care unit.

The older patients with a lower count of lymphocyte and platelet suffered more severe disease and stay in a longer hospital $[5,10]$.

The possible cause of platelet changes in COVID19 patients may be the direct invasion of the hematopoietic cells or bone marrow stromal cells by the corona virus, leading to hematopoietic inhibition, or because of the lung damage, given that the lung is the organ in which mature megakaryocytic release platelets $[11,12]$.

Alongside with thrombocytopenia, lymphopenia is the most common hematological sign during COVID. Qu et Al found that patients with lower lymphocyte levels at the first diagnosis are more seriously ill, and the progressive decline in lymphocyte proportion indicates a poor prognosis [10].

This finding supports the use of lymphocytes and platelets counts as markers that may flag patients with COVID-19 more likely to require admission to the hospital. However, a lymphopenia or thrombocytopenia are not sufficiently sensitive or specific to predict COVID disease status (sensitivity of 76\%; 84\% and specificity of $66 \%$; $40 \%$ respectively in our dataset).

In term of leukocyte, authors have found that patients with severe COVID can be leucopenic and others found them to be hyper leucocytic [5,13]. Liu et al. monitored the kinetic changes of lymphocyte subsets and cytokine profiles in COVID-19 patients, and showed that the total number of white blood cells in peripheral blood is normal or decreases in the early stages of the disease. Driven by lymphopenia, especially T cells CD4 and CD8. The decrease reached its peak within the first week of the disease course, and then $\mathrm{T}$ cell numbers gradually increased during the second week and recovered to a comparable level to that of the mild patient in the third week. Besides that, there is an increase in the levels of circulating neutrophils over time in both severe and non-severe COVID-19 infections. What will normalize or increase the white blood cell count[14].

This helps to understand why leukocytes and neutrophils were not significantly correlated with the severity of infection and suggests that the heterogeneity of our study sample regarding the timing of infection and the phase of immune response.

As a new type of inflammation index, PLR mainly reflects the level of systemic inflammation. Previous studies have confirmed that PLR is closely related to tumors, diabetes, coronary heart disease, and connective tissue diseases, and the increase of PLR is related to tumor size, lymph node infiltration, distant metastasis and prognosis, and can be used as a potential inflammatory indicator for the clinical diagnosis of community-acquired pneumonia [10].The advantage 
of PLR selection is that it reflects both aggregation and inflammatory pathways, and may be more valuable in predicting the severity of the cytokine storm than platelet or lymphocyte counts alone. According to the current clinical and relevant data, the PLR is positively correlated with the severity of the inflammation, the disease progression and the prognosis of COVID-19 patients.

Surprisingly, neutrophils, NLR, and d-NLR were not significantly related with disease severity, despite the fact that numerous authors have noted their utility in monitoring COVID disease [5,13-15]. In fact, We noted in this study that the mean of the NLR value in patients classified in the non-severe group is $12.57 \pm 14.14$, while most authors suggest a cut-off around 3-4 [13]. This suggests a lack of categorization of the study subgroups and that there were patients whose disease severity was underestimated.

In our study, PT values in severe COVID-19 patients were found to be lower, whereas those of D-dimer were found to be higher than in non-severe COVID patients , thus confirming earlier similar findings $[8,16]$. Even more importantly, the gradual progression of disease severity is mirrored by increasing values of D-dimer $[16,17]$.

Our study has a number of limitations. It is a retrospective, observational analysis of a narrow geographic region during a specific period of time. Then the experimental data is limited and many Biochemical test such as C-reactive protein, Procalcitonin, IL6 were missing from the analysis. And finally, it is preferable to carry out monitoring of the various biological examinations over time in order to better characterize the evolution and specify the prognostic value of each biological parameter.

\section{Conclusion}

In summary, thrombocytopenia, lymphopenia, increased PLR and D-dimers are abnormalities often encountered during severe Covid and can be used as a predictive marker for the severity of the disease. Further studies, with short and longterm follow-up and monitoring, is needed to establish low-cost biological assays like NLR and PLR as prognosis markers and complications predictors.

\section{Compliance with ethical standards}

\section{Acknowledgments}

The authors would like to thank the healthcare workers and laboratory teams at Avicenna military Hospital in Marrakech and Dr Ed-dyb for proofreading this manuscript.

\section{Disclosure of conflict of interest}

All authors declare that they have no conflicts of interest.

\section{Statement of informed consent}

The imperative of informed consent was waived in light of the anonymous, retrospective, and observational character of this study.

\section{References}

[1] Henry BM, Oliveira MHS de, Benoit S, Plebani M, Lippi G. Hematologic, biochemical and immune biomarker abnormalities associated with severe illness and mortality in coronavirus disease 2019 (COVID-19): a metaanalysis. ClinChem Lab Med CCLM. 2020; 58: 1021-8.

[2] Bhaskar S, Sinha A, Banach M, Mittoo S, Weissert R, Kass JS, et al. Cytokine Storm in COVID-19Immunopathological Mechanisms, Clinical Considerations, and Therapeutic Approaches: The REPROGRAM Consortium Position Paper. Front Immunol. 2020; 11: 1648.

[3] Chen G, Wu D, Guo W, Cao Y, Huang D, Wang H, et al. Clinical and immunological features of severe and moderate coronavirus disease 2019. J Clin Invest. 2020; 130: 2620-9.

[4] Guan W, Ni Z, Hu Y, Liang W, Ou C, He J, et al. Clinical Characteristics of Coronavirus Disease 2019 in China. N Engl J Med. 2020; 382: 1708-20. 
[5] Chandler CM, Reid MC, Cherian S, Sabath DE, Edlefsen KL. Comparison of Blood Counts and Markers of Inflammation and Coagulation in Patients With and Without COVID-19 Presenting to the Emergency Department in Seattle, WA. Am J ClinPathol. 2021; 156: 185-97.

[6] Ying H-Q, Deng Q-W, He B-S, Pan Y-Q, Wang F, Sun H-L, et al. The prognostic value of preoperative NLR, d-NLR, PLR and LMR for predicting clinical outcome in surgical colorectal cancer patients. Med Oncol Northwood Lond Engl. 2014; 31: 305.

[7] Lagunas-Rangel FA. Neutrophil-to-lymphocyte ratio and lymphocyte-to-C-reactive protein ratio in patients with severe coronavirus disease 2019 (COVID-19): A meta-analysis. J Med Virol. 2020; 92: 1733-4.

[8] Han H, Yang L, Liu R, Liu F, Wu K-L, Li J, et al. Prominent changes in blood coagulation of patients with SARS-CoV2 infection. ClinChem Lab Med. 2020; 58: 1116-20.

[9] Tjendra Y, Al Mana AF, Espejo AP, Akgun Y, Millan NC, Gomez-Fernandez C, et al. Predicting Disease Severity and Outcome in COVID-19 Patients: A Review of Multiple Biomarkers. Arch Pathol Lab Med. 2020; 144: 1465-74.

[10] Qu R, Ling Y, Zhang Y, Wei L, Chen X, Li X, et al. Platelet-to-lymphocyte ratio is associated with prognosis in patients with coronavirus disease-19. J Med Virol. 2020.

[11] Pilaczyńska-Cemel M, Gołda R, Dąbrowska A, Przybylski G. Analysis of the level of selected parameters of inflammation, circulating immune complexes, and related indicators (neutrophil/lymphocyte, platelet/lymphocyte, CRP/CIC) in patients with obstructive diseases. Cent-Eur J Immunol. 2019; 44: $292-8$.

[12] Eickmann M, Gravemann U, Handke W, Tolksdorf F, Reichenberg S, Müller TH, et al. Inactivation of three emerging viruses - severe acute respiratory syndrome coronavirus, Crimean-Congo haemorrhagic fever virus and Nipah virus - in platelet concentrates by ultraviolet C light and in plasma by methylene blue plus visible light. Vox Sang. 2020; 115: 146-51.

[13] Yang A-P, Liu J, Tao W, Li H. The diagnostic and predictive role of NLR, d-NLR and PLR in COVID-19 patients. IntImmunopharmacol. 2020; 84: 106504.

[14] Liu J, Li S, Liu J, Liang B, Wang X, Wang H, et al. Longitudinal characteristics of lymphocyte responses and cytokine profiles in the peripheral blood of SARS-CoV-2 infected patients. Biomedicine. 2020; 55: 102763.

[15] Liu Y, Du X, Chen J, Jin Y, Peng L, Wang HHX, et al. Neutrophil-to-lymphocyte ratio as an independent risk factor for mortality in hospitalized patients with COVID-19. J Infect. 2020; 81: e6-12.

[16] Tang N, Bai H, Chen X, Gong J, Li D, Sun Z. Anticoagulant treatment is associated with decreased mortality in severe coronavirus disease 2019 patients with coagulopathy. J ThrombHaemost. 2020; 18: 1094-9.

[17] Lippi G, Plebani M. Laboratory abnormalities in patients with COVID-2019 infection. ClinChem Lab Med CCLM. 2020; 58: 1131-4. 